\title{
Medullary thyroid carcinoma relapse reversed with dichloroacetate: A case report
}

\author{
DANA FLAVIN \\ Foundation for Collaborative Medicine and Research, Greenwich, CT, USA \\ and AMC/co Klinik im Alpenpark, Ringsee, Germany
}

Received April 7, 2010; Accepted June 15, 2010

DOI: 10.3892/ol_00000158

\begin{abstract}
A 51-year-old male patient diagnosed with medullary thyroid carcinoma (MTC) in 2001, with progression to lung metastases following adriamycin therapy, was then successfully treated with dimethyltriazenoimidazole carboximide. He remained in partial remission for 7 years following numerous chemotherapy attempts to induce partial remission. In October 2008, the patient, then 58 years old, relapsed with numerous tumors throughout his central body. On December 1 , 2008, the tumor marker for MTC, calcitonin, was at 38,611 pg/ $\mathrm{ml}$, i.e., much higher than the norm of $<20 \mathrm{pg} / \mathrm{ml}$. Since all other chemotherapy attempts had failed, he was ineligible for any new studies. Subsequently, the patient was immediately started on $10 \mathrm{mg} / \mathrm{kg}$ of dichloroacetate (DCA). By April 2009 , the calcitonin level was reduced to $2,000 \mathrm{pg} / \mathrm{ml}$. In May 2009, a new positron emission tomography showed a dramatic reduction in all tumor locations. The patient presently remains in remission and continues receiving the same dosage of DCA, with his tumor marker remaining stable in laboratory data since November 2009.
\end{abstract}

\section{Introduction}

Medullary thyroid carcinoma (MTC) is a rare calcitonin-producing neuroendocrine tumor arising from neural crest-derived parafollicular C cells (calcitonin-producing cells) of the thyroid gland (1), a component of multiple endocrine neoplasia type 2 or MEN2 syndromes (2), accounting for approximately $4 \%$ of all thyroid cancers. Although 25-30\% of MTC cases are inherited disorders, the remaining cases consist of sporadic forms of the disease (3). The 3-year survival rate of patients with differentiated thyroid cancer and radioactive iodine resistance is less than $50 \%$, with little response obtained from standard cytotoxic chemotherapies (4). In endocrine malignancies, such as thyroid carcinoma, the treatment of choice is surgery;

Correspondence to: Dr Dana Flavin, Foundation for Collaborative Medicine and Research, 24 Midwood Drive, Greenwich, CT, USA E-mail:dana@collmed.com

Key words: dichloroacetate, medullary thyroid carcinoma however, this approach is only successful in early stage disease. MTC is an incurable disease once metastases become unresectable (5). Treatment options for advanced endocrine malignancies remain unsatisfactory and are associated with poor patient prognoses. Cytotoxic chemotherapy and radiation therapy, which are associated with significant toxicity, often show only limited and transient efficacy (6). Tyrosine kinase inhibitors particularly effecting the RET (rearranged during transfection) proto-oncogene gain of function activity, such as with vandetanib, sorafenib and sunitinib, appear to be promising. However, the low rate of partial responses and the absence of complete responses in all of the various trials of monotherapy emphasize the need for novel and more effective single or combination of agents with acceptable toxicity (3).

\section{Case report}

This study presents a male MTC patient with lung metastases, who developed new tumors seven years after surgical intervention and successful chemotherapy with dimethyltriazenoimidazole carboximide (DTIC) and 5-fluorouracil. Initially diagnosed in 2001, the 51-year-old patient with MTC and lymph node involvement had a sporadic form of the disease, while his twin brother, who was also negative for any genetic factor, was disease-free. The patient was treated again in 2004 with a regimen of carboplatin and daunoxome, and again later that year with a depot of sandostatin (carbo-Iressasomatostatin) and etoposide plus carboplatin, to reduce his calcitonin levels from $13,280 \mathrm{pg} / \mathrm{ml}$; however, they increased to $20,074 \mathrm{pg} / \mathrm{ml}$. In 2005 , the regimen was changed to carboplatin, Iressa, Sandostatin and Zometa. In the Fall of 2005, he was administered samarian-153 for pain caused by bone metastases. In 2006, further chemotherapy of mitomycin C and cisplatin was administered, while he continued on Tarceva and Sandostatin. The calcitonin levels remained elevated.

In October 2008, the patient began to complain of diarrhea, and his tumor markers showed an increase in calcitonin to $25,843 \mathrm{pg} / \mathrm{ml}$ (norm $<20 \mathrm{pg} / \mathrm{ml}$ ). A positron emission tomography (PET; Siemens Ecat Exact 47) scan ordered that same October showed multiple new metastases in the thoracic area and mediastinum. The central chest cavity included more than five mediastinum multiple tumors and one large tumor anterior to the pericardium in the right mediastinal area with standardized uptake value (SUV >5). Additionally, 


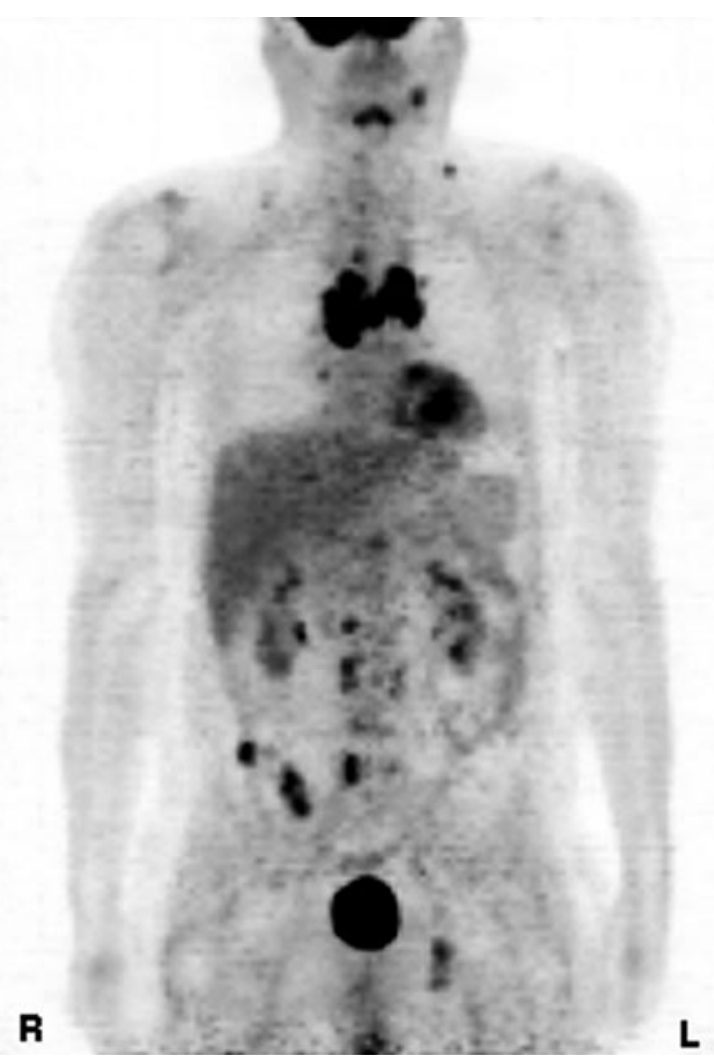

Figure 1. Positron emission tomography of the medullary thyroid cancer, October 2008

very small tumors in the vertebrae, including thoracic $\mathrm{T} 4$ and lumbar 3, 4 and 5 were noted. Other tumors were noted with an SUV of 5 in the os ileum and dorsal para-iliosacral region. Small tumors with an SUV of 3 were noted in the os acetabulum and the ascending ischium. Moreover, small tumors of an SUV of 2 were noted in the retroclavicular areas as well as more small tumors behind the sternocleidomastoid. By December 2008, the tumor marker calcitonin had increased to $38,611 \mathrm{pg} / \mathrm{ml}$.

The patient was ineligible for any of the new tyrosine kinase inhibitor studies provided and was given a poor prognosis as all known chemotherapies had failed. On December 1, 2008, the patient was placed on $10 \mathrm{mg} / \mathrm{kg}$ dichloroacetate (DCA) in an attempt to inhibit growth of the tumors and potentially obtain a reversal. Thiamin capsules at $750 \mathrm{mg}$ were supplemented to reduce any nerve toxicity reported from the DCA treatment (7).

In February 2009, a decrease in the calcitonin tumor marker to $8,655 \mathrm{pg} / \mathrm{ml}$ (norm $<20 \mathrm{pg} / \mathrm{ml}$ ) was first noted. A further reduction in calcitonin to $2,000 \mathrm{pg} / \mathrm{ml}$ was noted two months later. In May 2009, after 5 months of DCA administration, the PET scan showed a noteworthy reduction in tumor numbers and sizes. The lumbar metastases and tumors in the pelvic area were markedly reduced compared to October 2008, as were the thoracic area tumors, with only a small shadow in the T4 region hinting at the location of the metastasis originally noted in the initial PET in 2008. The lymph node metastases were very small compared to the earlier exam, and central necrosis had increased in the mediastinal tumor.

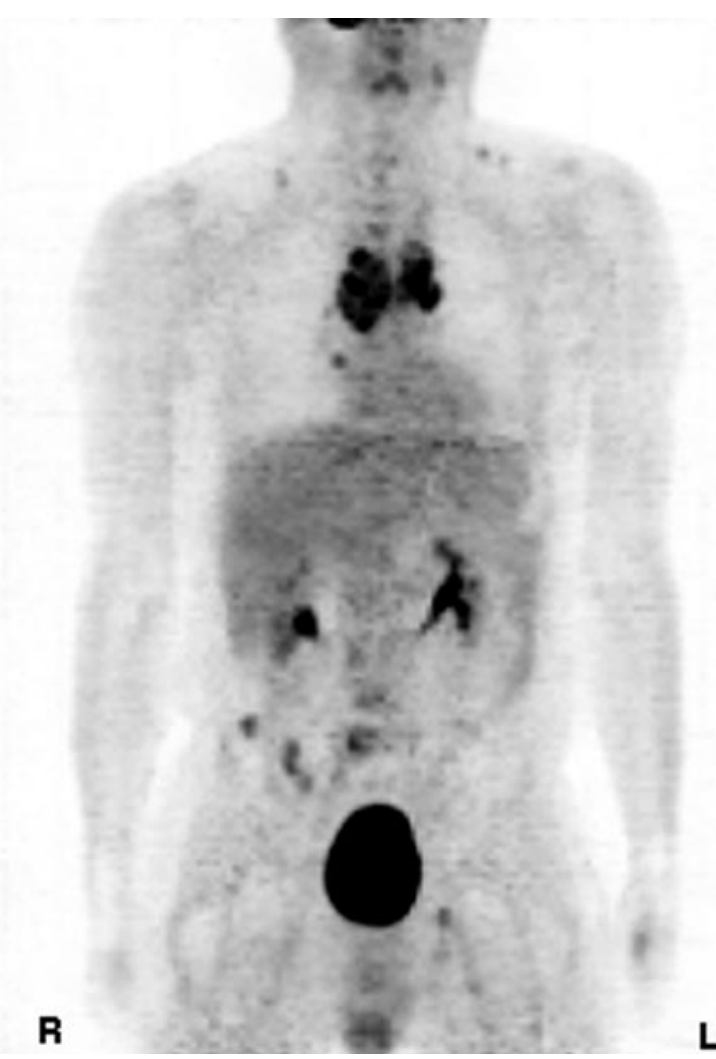

Figure 2. Positron emission tomography of the medullary thyroid cancer after dichloroacetate treatment, May 2009.

\section{Discussion}

MTC is a rare, but challenging malignancy (8). Poor prognostic factors include age over 50 years, male gender and distant spread (metastasis). The 10-year survival is only $20 \%$ for patients with distant metastases. The blood calcitonin levels are measured to determine the growth of MTC in patients with a positive test defined as a basal serum calcitonin more than or equal to $50 \mathrm{pg} / \mathrm{ml}(9)$. In this case, the patient had a poor prognosis since he was over 50 years of age, male and with distant metastases. In 2001, the patient was diagnosed and first treated with DTIC, after adriamycin had resulted in a spread of the cancer with lung metastases. Following remission from his lung metastases, his calcitonin levels fluctuated between 7,000 and $13,000 \mathrm{pg} / \mathrm{ml}$ (norm $<20$ $\mathrm{pg} / \mathrm{ml}$ ). From 2004 until 2007, the calcitonin levels gradually increased to over $20,000 \mathrm{pg} / \mathrm{ml}$ despite numerous attempts to reduce the levels with various chemotherapies, demonstrating a minimal effect on the inhibition of the cancer progression. By October 2008, the tumors had spread throughout the bone and mediastinal areas, and the calcitonin tumor marker level reached over $38,000 \mathrm{pg} / \mathrm{ml}$. PET scans correlated with the excess calcitonin levels, indicating a very dire prognosis. In our case of MTC, the use of DCA combined with $750 \mathrm{mg}$ of thiamin to counteract any nerve toxicity resulted in a marked reversal of the tumors and tumor marker levels over a 5-month period. Although the patient's gastrointestinal system returned to normal within the first few weeks of the DCA therapy, a few months elapsed before a reduction in the calcitonin levels and the general feeling of fatigue was noted. 
The extensive number of tumors, the elevated calcitonin levels, the increased adverse symptoms associated with treatment modalities, the lack of chemotherapy responses and the ineligibility for experimental tyrosine kinase inhibitor studies, warranted the use of DCA. DCA has been successfully used in children with congenital lactic acidosis since 1988 (10), and is a chemical currently tested oncologically in Canada.

Notably, numerous tumor cells exhibit an increase in lactic acid from the preferential use of glycolysis instead of glucose oxidation to generate adenosine triphosphate (ATP) even in the presence of oxygen. This phenomenon is known as aerobic glycolysis or the 'Warburg effect' (11), which results in pyruvate being directed to lactic acid instead of into the mitochondria for ATP generation in the tricarboxylic acid (TCA) cycle. DCA allows for the reactivation of pyruvate dehydrogenase, a gate-keeping enzyme for the entry of pyruvate into the mitochondrial TCA by blocking pyruvate dehydrogenase kinase (12). This blockage in glycolysis shifts the tumor cells back to the utilization of glucose oxidation; the normal aerobic metabolism noted in healthy cells that leads to apoptosis (13).

The successful treatment of this MTC patient is only a preliminary result, and further research should be conducted to confirm our finding in other MTC patients who have exhausted chemotherapeutic treament modalities. The results noted in this case may also indicate a potential application for DCA in this form of cancer when other therapies fail, at least until novel therapies are developed for patients with only a $20 \%$ chance of survival over a 10 -year period.

In conclusion, we presented an MTC patient with multiple metastases and a very poor prognosis after seven years of chemotherapy whose relapse was reversed with DCA treatment. This proposes a mechanism that may open new doors for the future development of novel cancer therapies.

The patient is presently being maintained on DCA for an indefinite period given its positive results of remission. As of November 2009, he is in remission according to his tumor marker levels. Any changes in the tumor marker level will indicate resistance to this therapy. Other MTC patients may be considered for this therapeutic approach in the future, should it be deemed necessary. To conclude, DCA inhibited tumor progression and tumor marker levels. Additionally, the mechanism of DCA, which involves reversing glycolysis, appears to be an important target for investigation as a future therapeutic approach.

\section{Acknowledgements}

I would like to thank Marc Ramos and Jimmy $\mathrm{Xu}$ for the scientific research assistance. This study did not receive any specific grant from any funding agency in the public, commercial or non-profit sector.

\section{References}

1. Santarpia L, Ye L and Gagel RF: Beyond RET: potential therapeutic approaches for advanced and metastatic medullary thyroid carcinoma. J Intern Med 266: 99-113, 2009.

2. Cuccuru G: Cellular effects and antitumor activity of RET inhibitor RPI-1 on MEN2A-associated medullary thyroid carcinoma. J Natl Cancer Inst 96: 1006-1014, 2004.

3. Cakir M and Grossman A: Medullary thyroid cancer: molecular biology and novel molecular therapies. Neuroendocrinology 90 : 323-348, 2009.

4. Higgins MJ, Forastiere A and Marur S: New directions in the systemic treatment of metastatic thyroid cancer. Oncology 23: 768-775, 2009.

5. Sugawara M, Geffner DL, Martinez D and Hershman JM: Novel treatment of medullary thyroid cancer. Curr Opin Endocrinol Diabetes Obes 16: 367-372, 2009.

6. Fassnacht M, Kreissl MC, Weissmann D and Allolio B: New targets and therapeutic approaches for endocrine malignancies. Pharmacol Ther 123: 117-141, 2009.

7. Spruijt L, Naviaux R, McGowan KA, et al: Nerve conduction changes in patients with mitochondrial diseases treated with dichloroacetate. Muscle Nerve 24: 916-924, 2001.

8. American Thyroid Association Guidelines Task Force: Medullary thyroid cancer: Management Guidelines of the American Thyroid Association. Thyroid 19: 565-612, 2009.

9. Cheung K: Calcitonin measurement in the evaluation of thyroid nodules in the United States: a cost-effectiveness and decision analysis. Clin Endocrinol Metab 93: 2173-2180, 2008.

10. Stacpoole PW, Lorenz A, Thomas R and Harman E: Dichloroacetate in the treatment of lactic acidosis. Ann Intern Med 108: 58-63, 1988.

11. Warburg O, Wind F and Negelein E: Uber den stoffwechsel von Tumoren im Korper. Klin Woch 5: 829-832, 1926.

12. Stacpoole PW, Henderson GN, Yan Z and James MO: Clinical pharmacology and toxicology of dichloroacetate. Environ Health Perspect 106: 989-994, 1998.

13. Bonnet S, Archer SL, Allalunis-Turner J, et al: A mitochondria- $\mathrm{K}^{+}$channel axis is suppressed in cancer and its normalization promotes apoptosis and inhibits cancer growth. Cancer Cell 11: 37-57, 2007. 